\title{
Multiple scattering causes the low energy- low angle constant wavelength topographical instability of argon ion bombarded silicon surfaces
}

\section{Citation}

Madi, Charbel S., and Michael J. Aziz. 2012. "Multiple Scattering Causes the Low Energy-low Angle Constant Wavelength Topographical Instability of Argon Ion Bombarded Silicon Surfaces." Applied Surface Science 258, no. 9: 4112-4115. doi:10.1016/j.apsusc.2011.07.143.

\section{Published Version}

doi:10.1016/j.apsusc.2011.07.143

\section{Permanent link}

http://nrs.harvard.edu/urn-3:HUL.InstRepos:27417436

\section{Terms of Use}

This article was downloaded from Harvard University's DASH repository, and is made available under the terms and conditions applicable to Open Access Policy Articles, as set forth at http:// nrs.harvard.edu/urn-3:HUL.InstRepos:dash.current.terms-of-use\#OAP

\section{Share Your Story}

The Harvard community has made this article openly available.

Please share how this access benefits you. Submit a story.

\section{Accessibility}




\title{
Multiple Scattering Causes the Low Energy-Low Angle Constant Wavelength Topographical Instability of Argon Ion Bombarded Silicon surfaces
}

Charbel S. Madi and Michael J. Aziz, Harvard School of Engineering and Applied Sciences, Cambridge MA 02138 USA

\begin{abstract}
We show that holes and perpendicular mode ripples that are generated at low argon ion beam energies and incidence angles on room temperature silicon targets (C. S. Madi et al, Phys. Rev. Lett. 101, 246102 (2008) and C. S. Madi et al, J. Phys. Condens. Matter 21, 224010 (2009)) are caused by multiple scattering events from the impingement of the primary ion beam on adjacent silicon shields. We show that in a geometry that minimizes these multiple scattering events, only ultra-smooth stable silicon surfaces are for incidence angles $<50^{\circ}$ from normal. We present a revised topographical phase diagram of 250-1000 eV $\mathrm{Ar}^{+}$ion bombarded silicon surfaces in the linear regime of surface dynamics in the absence of secondary scattering effects. It is characterized only by a diverging wavelength phase transition from parallel mode ripples to a flat stable surface as the incidence angle falls below about $50^{\circ}$ from normal incidence, and a crossover to perpendicular mode ripples as the incidence angle crosses above about $80^{\circ}$.
\end{abstract}

\section{Introduction}

Pattern formation resulting from uniform ion irradiation of solid surfaces in the low energy regime (typically $10^{2}-10^{4} \mathrm{eV}$ ) where the energy loss is dominated by nuclear collision cascades has been the topic of continued experimental and theoretical investigations. Due to its simplicity, noble-gas ion irradiation of silicon has been extensively studied as a very promising system for experimental tests of theory: it is a monatomic system amenable to molecular dynamics simulation and its near-surface region is amorphous under ion bombardment, thereby minimizing the potentially confounding effects of disproportionation and crystallographic singularities [1]. However, despite its attractive attributes, the noble gas $\rightarrow$ silicon system has proven remarkably finicky, confounding researchers via interlaboratory irreproducibilities that now appear to be mainly the result of trace metal surface contamination [2]. In our experimental studies we have gone to great lengths to avoid trace impurity contamination of our silicon surfaces by, e.g., shielding all surfaces of the sample holder subject to direct ion irradiation with silicon [3].

We have recently shown both experimentally [4] and theoretically [5] that the topographical pattern-forming instability is determined by the effects of ion impact-induced prompt atomic redistribution and that sputter erosion - the erstwhile consensus predominant cause - is so much smaller an effect that it is essentially irrelevant, except possibly for the most grazing angles. We have also presented a parameter-free theory [5] that proposes a replacement of the erosion-based erstwhile paradigm with a new paradigm based on the crater function - the change in surface topography in the vicinity of an impact, averaged over many impacts. This 
theory, whose predictions are worked out for $250 \mathrm{eV} \mathrm{Ar}^{+}$impacts on amorphous silicon, reproduces the magnitude of the experimental wavelength as well as the experimental observation of a diverging-wavelength bifurcation - a phase transition from a rippled surface to a flat, stable surface - as the angle $\theta$ from normal incidence is reduced toward $\sim 45^{\circ}$ from higher angle. However, the theory does not reproduce the experimental observation of a constant-wavelength bifurcation from a flat surface to a rippled surface as the incidence angle is further decreased past $20^{\circ}[3,6]$. On the contrary, the theory predicts that a flat surface is maximally stable as normal incidence is approached.

In this paper, we show experimentally that the origin of the constant wavelength bifurcation exhibited by silicon at low ion beam incidence angle and energy is a multiple scattering artifact of either scattered argon or recoiled silicon impinging upon the target surface.

\section{Experiment}

\subsection{Procedures}

We performed argon ion irradiation experiments on $\operatorname{Si}(001)$ targets (p type, $1-10 \Omega \mathrm{cm}$ ) in a high vacuum chamber (base pressure $2 \times 10^{-8}$ Torr at room temperature) with the projected ion beam direction along the [110] crystallographic direction. The argon ions were generated using a $3 \mathrm{~cm}$ RF source with graphite accelerating grids. The ion flux from the source was $0.57 \mathrm{~mA} \mathrm{~cm}^{-2}$ in the plane perpendicular to the ion beam. The beam divergence was roughly $5^{\circ}$ and the distance to the target was approximately $15 \mathrm{~cm}$. To vary the incidence angle, targets were fixed, using melted indium (with no indium exposed to the ion beam), onto graphite wedges of various angles which were shielded everywhere from the ion beam by $\mathrm{Si}$ wafer shields. Thus, only silicon was exposed to the direct ion beam and contamination induced dynamics are suppressed. After irradiation, atomic force microscopy (AFM) images were obtained using an Asylum MFP-3D Stand Alone (MFP-3D-SA) system in tapping mode. During the course of these studies we noticed the sensitivity of pattern formation on $\mathrm{Ar}^{+}$ion-sputtered silicon surfaces to the detailed configuration with which the Si targets are fixed to the wedges of various inclinations within the vacuum chamber.

\subsection{Experimental Results}

In Figure 1(a) and 1(c), we show two different implemented geometries for normal incidence $\mathrm{Ar}^{+}$ion bombardment of a silicon target. In Figure 1(a), the ion gun is mounted at an angle $\alpha$ with respect to the surface normal of the silicon-shielded target holder, with the Si target fixed on a wedge with the same inclination angle $\alpha$. The resulting target surface topograph induced by normal incidence ion bombardment is shown in Figure 1(b): an array of holes with characteristic length scale of about $70 \mathrm{~nm}$ is distributed across the entire $1 \times 1 \mathrm{~cm}^{2} \mathrm{Si}$ surface. Figure 1(c) shows normal incidence $\mathrm{Ar}^{+}$ion bombardment of a Si target that is being fixed using melted indium to a wedge-free holder with the ion gun mounted along the target surface normal. The corresponding surface topography is featureless and smooth as shown in Figure 1(d). Hence, it appears likely that higher order collision effects such as secondary collisions by scattered argon and redeposition of sputtered silicon from the shields onto the 
target surface are directly responsible for the generation of the hole structure. Analysis of these complex processes would require knowledge of the scattered angular and energy distributions of primary ions as well as of the silicon atoms sputtered from the shields.

The results shown in Figure 2 further confirm our claim that pattern formation at normal incidence is due to secondary collisions in the target following primary collisions in the silicon shielding with line of sight to the target surface. In Figure 2 (a), we observe that as the distance $x$ in the target from the nearest point of the silicon shield increases, the observed topography changes from perpendicular mode ripples to flat and smooth for $x \geq 15 \mathrm{~mm}$. Figure 2(e) is consistent with the surface morphology of Figure 1(d) of wedge-free normal incidence $\mathrm{Ar}^{+}$ion beam sputtering.

In Figure 3, we present a phase diagram of patterns formed on $\mathrm{Ar}^{+}$ion bombarded silicon surfaces in the linear regime of surface dynamics in the absence of secondary scattering effects. The two control parameters are ion beam energy $E$ and angle $\theta$ from normal incidence. At all $\theta<47.5^{\circ}$, we observe ultra-smooth stable surfaces across all ion energies $250 \mathrm{eV} \leq E \leq 1000 \mathrm{eV}$, whereas at incidence angles of $50^{\circ} \leq \theta \leq 80^{\circ}$ we observe parallel mode ripples (wavevector parallel to the projected ion beam on the surface) characterized by a diverging wavelength as $\theta$ approaches $50^{\circ}$ from above. Finally, at sufficiently high angles $\theta$ $\approx 85^{\circ}$ we observe perpendicular mode ripples characterized by their wavevector being perpendicular to the ion beam. 


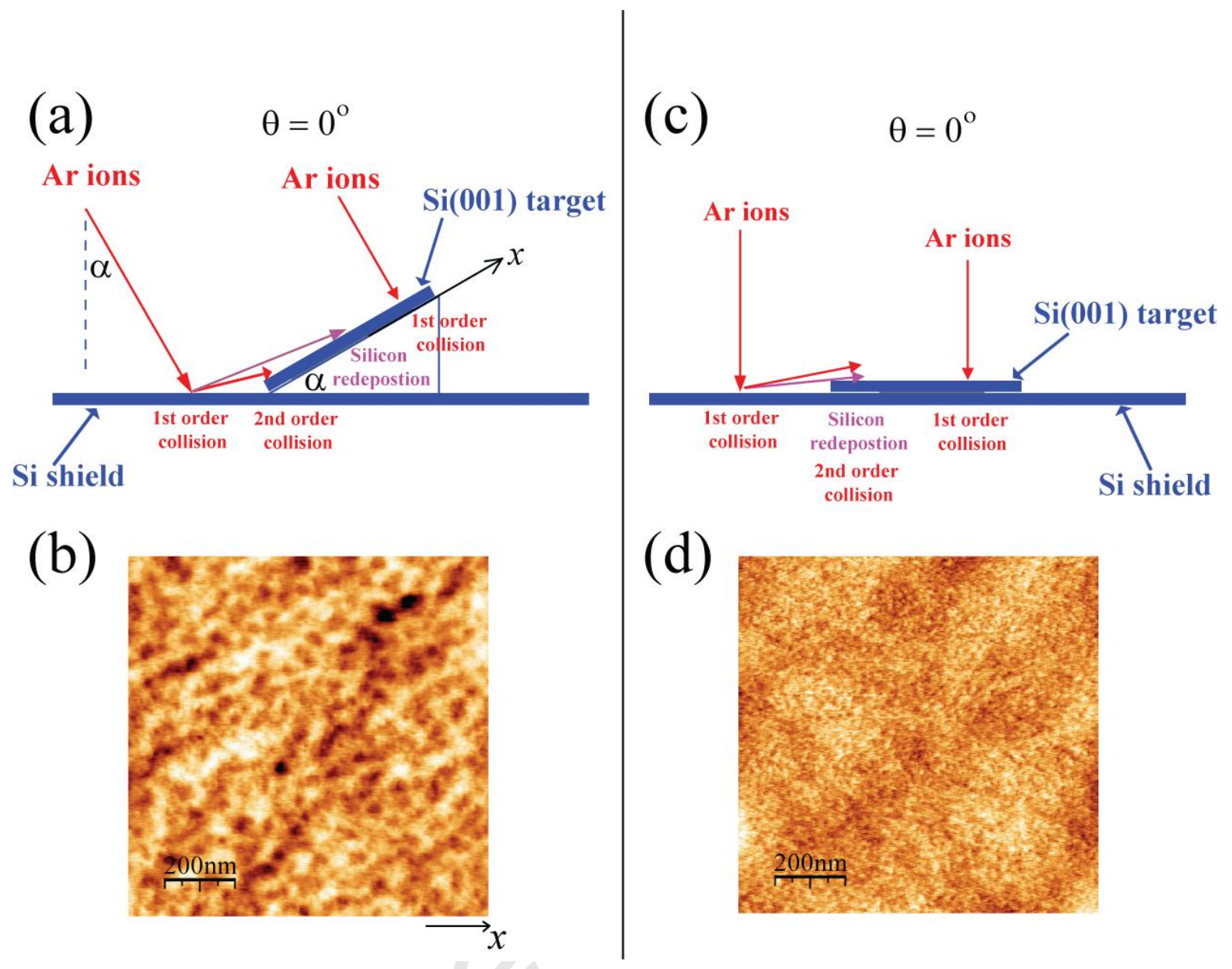

Figure 1. Effect on surface topography of secondary collisions in the $\mathrm{Si}(001)$ target following primary collisions at the silicon shielding with line of sight to the target surface. Figure 1(a) and 1(c) represent two different implemented geometries for normal incidence $\mathrm{Ar}^{+}$ion bombardment of a $\mathrm{Si}(001)$ target. Both AFM topographs in Figure 1(b) and Figure 1(d) are generated under identical experimental conditions of ion flux of $3.5 \times 10^{15}$ ions $/ \mathrm{cm}^{2} / \mathrm{sec}$, fluence of $4.5 \times 10^{18}$ ions $/ \mathrm{cm}^{2}$, ion beam energy of $250 \mathrm{eV}$, and normal ion beam incidence angle. The AFM topograph scan size is $1 \mu \mathrm{m} \times 1 \mu \mathrm{m}$. The vertical range is $2.5 \mathrm{~nm}$ in (b) and $1 \mathrm{~nm}$ in (d). 
(a)
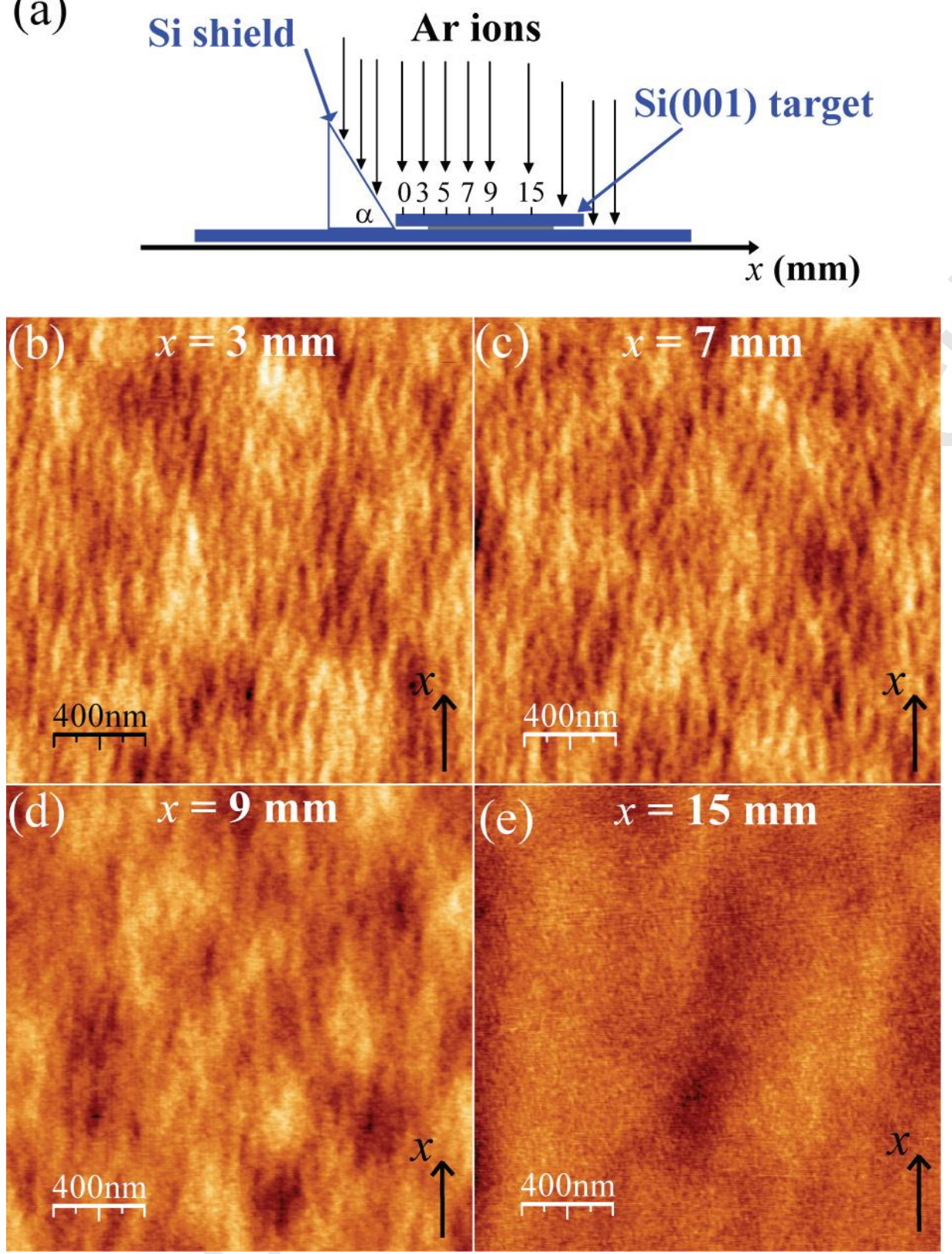

Figure 2. Effect on surface topography of secondary collisions in the $\mathrm{Si}(001)$ target following primary collisions at the silicon shielding with line of sight to the target surface. As distance $x$ from the edge of the wedge with angle $\alpha=60^{\circ}$ is increased, perpendicular mode ripples give way to a flat stable surface. The AFM topograph scan size is $2 \mu \mathrm{m} \times 2 \mu \mathrm{m}$. The vertical range is $3 \mathrm{~nm}$ in (b)-(d) and $2 \mathrm{~nm}$ in (e). The AFM topographs are obtained following irradiation with $250 \mathrm{eV} \mathrm{Ar}^{+}$at room temperature and fluence of $9.5 \times 10^{18}$ ions $/ \mathrm{cm}^{2}$. 


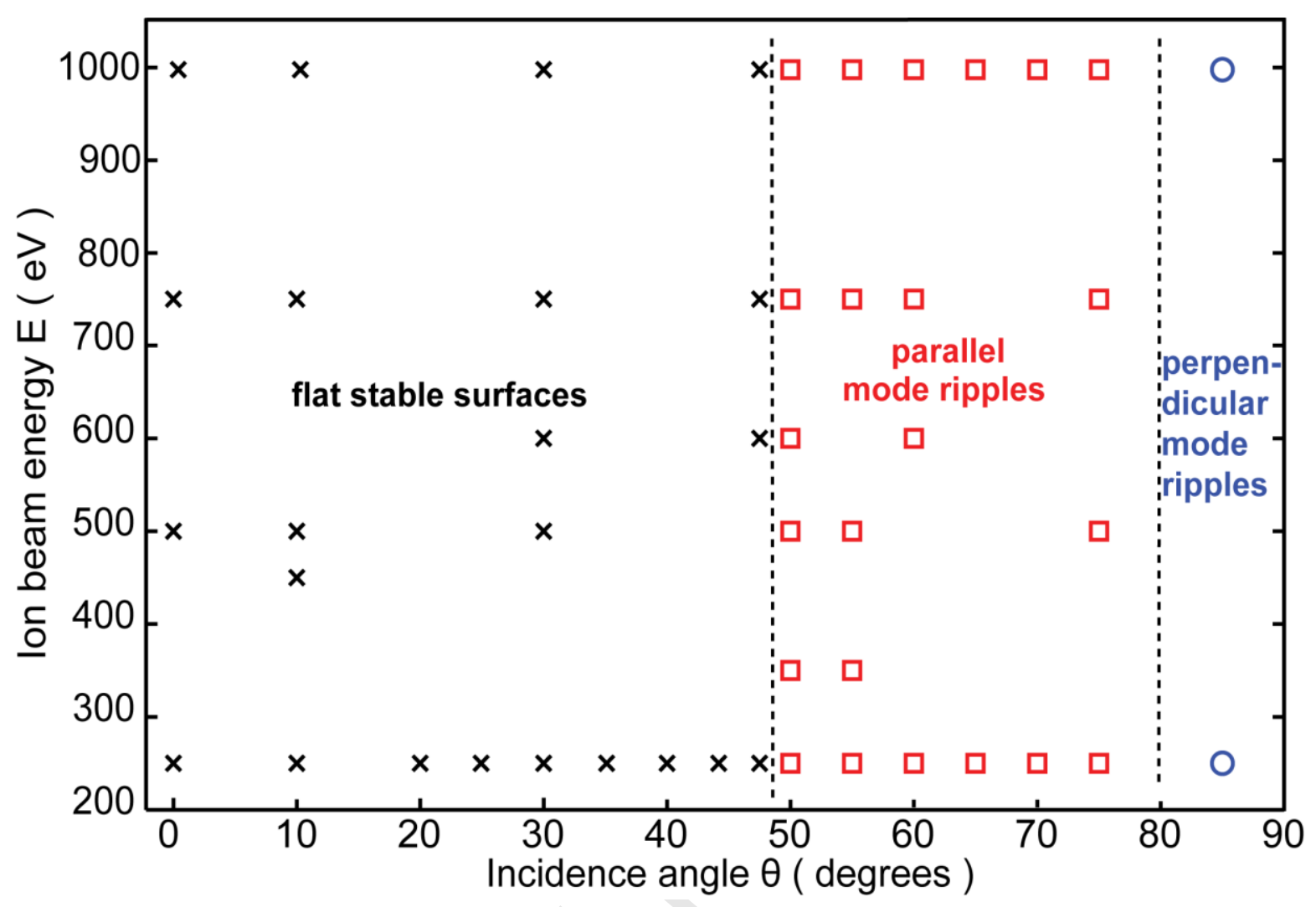

Figure 3. Phase diagram of pattern formation of $\mathrm{Ar}^{+}$ion beam sputtering of nominally room temperature $\mathrm{Si}(001)$ in the linear regime of surface dynamics in the absence of secondary scattering effects. $\times$ : flat; $\square$ : parallel mode ripples; $\bigcirc$ : perpendicular mode ripples. Fluence is $3.8 \times 10^{18} \mathrm{Ar}^{+} \mathrm{cm}^{-2}$ for flat stable surfaces and $3.2 \times 10^{17} \mathrm{Ar}^{+} \mathrm{cm}^{-2}$ for parallel and perpendicular mode ripples at $\theta \geq 50^{\circ}$.

\section{Conclusions}

The results presented here lead to the inescapable conclusion that the instability that we previously identified in the low-energy, low-angle corner of the $\mathrm{Ar}^{+} \rightarrow \mathrm{Si}$ morphology phase diagram $[3,6]$ is due to multiple scattering effects involving irradiation of silicon shields simultaneously with the silicon target. The phase diagram appearing in Figure 3 replaces the one we reported previously.

\section{Acknowledgments}

This research was supported by the U.S. Department of Energy under grant DE-FG02-06ER46335. We thank Professor Scott Norris of Southern Methodist University for helpful discussions.

\section{Literature Cited}


${ }^{1}$ W.L. Chan and E. Chason, J. Appl. Phys. 101, 121301 (2007); M. Kalff, G. Comsa, and T. Michely, Surface Science 486, 103 (2001).

${ }^{2}$ G. Ozaydin, A.S. Ozcan, Y.Y. Wang, K.F. Ludwig, H. Zhou, R.L. Headrick, and D.P. Siddons, Appl. Phys. Lett. 87, 163104 (2005); G. Ozaydin, K.F. Ludwig, H. Zhou, and R.L. Headrick, J. Vac. Sci. Technol. B 26, 551 (2008); S. Macko, F. Frost, B. Ziberi, D.F. Forster, and T. Michely, Nanotechnology 21, 085301 (9 pages) (2010).

${ }^{3}$ C.S. Madi, H.B. George, and M.J. Aziz, Journal of Physics-Condensed Matter 21, 224010 (2009).

${ }^{4}$ C.S. Madi, E. Anzenberg, K.F. Ludwig, and M.J. Aziz, Phys Rev Lett 106, 066101 (2011).

${ }^{5}$ S.A. Norris, J. Samela, L. Bukonte, M. Backman, F. Djurabekova, K. Nordlund, C.S. Madi, M.P. Brenner, and M.J. Aziz, Nature Communications 2, 276 (2011).

${ }^{6}$ C.S. Madi, B. Davidovitch, H.B. George, S.A. Norris, M.P. Brenner, and M.J. Aziz, Physical Review Letters 101, 246102 (2008). 